Modern Physics Letters A

Vol. 26, No. 16 (2011) 1243

(C) World Scientific Publishing Company

DOI: $10.1142 / \mathrm{S} 0217732311036024$

\title{
Erratum
}

\section{GEOMETRIZATION OF ELECTROMAGNETISM IN TETRAD-SPIN-CONNECTION GRAVITY}

\author{
[Mod. Phys. Lett. A, Vol. 24, No. 6 (2009) 431-442] \\ N. J. Popławski
}

On p. 439 , Eq. (42) should read

$$
2 \mathfrak{e} \gamma^{\mu} \psi_{\mid \mu}+\mathfrak{e} \gamma^{\mu} \Gamma_{\mu} \psi-\mathfrak{e} \Gamma_{\mu} \gamma^{\mu} \psi+\left(\mathfrak{e} \gamma^{\mu}\right)_{; \mu} \psi-2 \mathfrak{e} S_{\mu} \gamma^{\mu} \psi+2 i \mathfrak{e} m \psi=0
$$

